\title{
Tecnología asistencial y el cuidado enfermero
}

\author{
Olga Giovanna Valderrama Rios, ${ }^{1}$ Guillermina Valdivia Arce $^{2}$
}

Valderrama ROG, Valdivia AG. Tecnología asistencial y el cuidado enfermero. Cuid salud, enejun 2014; 1(1).

\section{RESUMEN}

El profesional de Enfermería enfrenta un desarrollo tecnológico en el cual el cuidado humano debe estar enmarcado bajo los parámetros de la bioética. Ese cuidado significa realizar acciones acompañadas de actitudes y comportamientos de cuidar. Enfermería tiene un compromiso con el cuidado, si bien utilizando la mejor tecnología, ésta no debe suplantar el lado humano, la persona es el verdadero centro del cuidado, lo que implica informar adecuadamente al paciente respetando sus valores. Entre tanto: ¿Estamos brindando cuidado humanizado a los pacientes? En el contexto asistencial: ¿Qué relación existe entre la tecnología y el cuidado humano? ¿Es posible dar un cuidado humano con base en el uso de la tecnología?.

Palabras clave: paciente crítico, enfermería, tecnología, humanizar, ética y bioética.
Valderrama ROG, Valdivia AG. Assistive technology and nurse care. Cuid salud, ene-jun 2014; 1(1).

\begin{abstract}
Professional Nursing feces a technological development which human care must be delimited under the parameter of bioethics. That care means to perform actions accompanied by attitudes and behaviors of caring. Nursing has a commitment, by using the best technology, but it should not replace the human side, the person is the true Center of caring, which means to inform properly to the patient respecting their values. Meanwhile: Are we providing humanized care to our patients? In the practice context: What is the relationship between technology and human care? Is it possible to provide humane care based on the use of technology?.
\end{abstract}

Keywords: critical patient, nursing, technology, humanize, ethics and bioethics.

\footnotetext{
${ }^{1}$ Licenciada enfermera. Magister. Especialista en cuidado intensivo neonatal. Docente asociada de la Facultad de Ciencias Médicas - Escuela Profesional de Enfermería - Universidad Nacional Santiago Antúnez de Mayolo. Ancash - Perú. ${ }^{2}$ Licenciada enfermera. Especialista en cuidados intensivos. Docente de la Escuela de Enfermería Padre Luis Tezza, afiliada a la Universidad Ricardo Palma, Lima-Perú.
} 


\section{INTRODUCCIÓN}

La tecnología tuvo su expansión en el contexto mundial desde fines del siglo XVIII hasta principios del siglo XIX. Y la Enfermería, como disciplina de las ciencias de la salud, ha sido influenciada por las tecnologías que dieron lugar a diferentes formas de cuidar, poniéndose de manifiesto la necesidad de integrar al cuidado enfermero diferentes tipos de tecnologías: tecnología blanda, blanda dura y dura. ${ }^{1}$

La tecnología blanda hace referencia a las relaciones, interacción, recepción, comunicación con el paciente y familia, además de la gestión de los servicios; la blanda dura, es una de las tecnologías más relevantes para el desarrollo de la Enfermería, remite al conocimiento bien estructurado, tal como lo es el proceso de enfermería, que ha permitido desarrollar un corpus de conocimiento propio, ha generado conocimientos nuevos y ha dado impulso a la investigación; finalmente, en la tecnología dura se destacan los equipos biomédicos como, la ventilación mecánica y las bombas infusoras; así también, se encuentra aquí la expresión tecnológica de la atención como un proceso y un producto: traducida en la construcción del conocimiento enfermero a través de la sistematización de técnicas y la organización de principios científicos sobre la base científica de los cuidados de enfermería. ${ }^{1}$ Destacando que cuidar es la interacción continua de un conjunto de instrumentos, técnicas, procesos y personas; todos componentes importantes en la relación enfermerapaciente.

En concordancia con Boykin y Schoenhofer, ${ }^{2}$ toda persona es un ser de cuidado en virtud de su propia humanidad, que vive y crece en el cuidado, el cual se fortalece en su relación con los otros, el cuidado engloba los aspectos científicos, humanistas, instrumentales y expresivos, como un conjunto de elementos, todos inseparables unos de otros en el acto de cuidar.

En este contexto, las enfermeras (os) deben considerar que cuidar es comprender con empatía, es sentir con el otro, visualizando al otro como un sujeto en sí mismo, recordando que representa la esencia de la profesión; sin embargo, muchas veces se otorga más importancia a la tecnología dura que forma parte del cuidado, dejando de lado la interacción, el acto y la escucha atenta. ${ }^{3}$ Este ensayo expone la opinión de las autoras sobre la base de tres argumentos, en los que se evidencia fundamentos teóricos humanísticos que permiten reflexionar de qué manera se brinda el cuidado enfermero a partir del uso de la tecnología y del poder de la técnica; aspectos que constituyen nuevas exigencias en la práctica del cuidado, que ha significado el desplazamiento de una mayor atención al seguimiento y control de procesos estandarizados y sistematizados, con deterioro de la interacción enfermera-paciente, de la relación comprensiva y afectuosa, pilar fundamental del cuidado enfermero. ${ }^{4}$

El profesional de Enfermería encara una serie de tensiones y conflictos, tornándose algunas veces en situaciones que le confunden y frustran, pues siente que la manera de cómo aborda el cuidado sólo se enfoca en la rutina de procedimientos y técnicas. Sobre todo, porque su eficiente desempeño profesional debe hacerlo en un tiempo determinado, aunque de este modo se aleja de lo esencial del cuidado, que es la existencia misma de la persona que cuida, alguien tan igual como ella o él, y que hace parte activa del acto de cuidar. Ante lo expuesto, se formulan las siguientes interrogantes como propósitos de la reflexión ¿Se está brindando cuidado humanizado a los pacientes? En el contexto asistencial: ¿Qué relación existe entre la tecnología y el cuidado humano? ¿Es posible dar un cuidado humano con base en el uso de la tecnología?

\section{PRIMER ARGUMENTO}

Ante la apremiante deshumanización del cuidado enfermero, tomando cuenta la gran reestructuración administrativa en los sistemas de salud del mundo, consecuencia de la globalización que visualiza la asistencia sanitaria como empresas competitivas, tenaces, eficientes y leales, enmarcados en valores técnicos-científicos por sobre la calidad y calidez del cuidado; siendo los pacientes un eslabón más en el mecanismo de la productividad, bajo el desarrollo de funciones que encasilla el cuidado en patrones estandarizados, dejando de lado la individualidad y la unicidad compleja del ser humano. Al ingresar al sistema sanitario, la persona enferma pierde su identidad y es reconocida apenas como parte de un grupo o un área "especializada", convirtiéndose en un valor numérico que se suma a las estadísticas y porcentajes en los hospitales. ${ }^{5}$ Realidad que pone en evidencia la necesidad del rescate del aspecto humano, espiritual y transpersonal del cuidado que brindan especialmente los profesionales de enfermería, tanto en la práctica clínica como en la administrativa. Aspecto clave en la forma actual de 
desarrollar y relacionarse con el cuidado de Enfermería, pues muchas veces se soslaya el centro del quehacer: la persona, el individuo, el usuario, el paciente, el cliente o como sea llamado el "ser humano".

Para Jean Watson, persona, es "un ser en el mundo, como una unidad de mente-cuerpo y espíritu, que experimenta y percibe conceptualmente el Gestalt, el locus de la existencia humana y el sujeto de cuidado de Enfermería". 6 La autora, citando a Peplau, explica que las bases de la Gestalt, que tiene como significado "integración", son el aquí y el ahora, lo obvio y la toma de consciencia, es decir, el "darse cuenta" desde una mirada existencialista; dado que el cuidado de enfermería no es un hecho único aislado, ni etéreo, sino que se da en contextos sociales, institucionales y en redes de relaciones que le otorgan un significado. Por esta razón, la enfermera como instrumento terapéutico y experto técnico debe tener conocimientos, clarificar sus valores y estar comprometida con el cuidado y para tal, utilizar todos los recursos disponibles y los avances tecnológicos que le ayuden a mantener la vida y preservar la dignidad del paciente. ${ }^{6}$ Entendiendo que cada encuentro enfermera-paciente es una oportunidad transformadora y dignificante por su esencial contenido humanístico: es una oportunidad de relación, madurez, aprendizaje y crecimiento mutuo.

Bajo el paradigma de la integración la enfermera ha orientado su visión hacia la persona, dirigiendo los cuidados a mantener la salud en todas sus dimensiones: física, mental y social. En ese sentido, compete a la enfermera evaluar las necesidades de ayuda de la persona considerando sus percepciones y su globalidad. Ariza y Caballero $^{4}$ citando a Meleis, refieren que el cuidado debe focalizarse en la situación presente utilizando los principios de la relación de ayuda, que significa "actuar con" la persona, a fin de responder a sus necesidades. En esta perspectiva, la enfermera planifica y evalúa sus acciones en función de las necesidades no satisfechas, visualizando a la persona como un todo formado por la suma de sus partes, las cuales están interrelacionadas desde sus componentes biológicos, psicológicos, culturales y espirituales. De ahí la expresión: la persona es un ser bio-psicosocio-cultural-espiritual, aunque muchas veces se deja de lado todos estos componentes. ${ }^{7}$

Pero ¿Por qué sucede esto? Existen varios factores que pueden interferir en esta visión. Como se destaca el estudio de Aguirre, ${ }^{8}$ quien hace referencia a la escasez de recursos humanos en los hospitales, la multifuncionalidad del profesional, entre otros, que producen insatisfacción laboral en las enfermeras. Condición que puede resultar peligrosa para el sistema de salud, puesto que atender a los seres humanos en forma descontenta puede traer consecuencias perjudiciales para la salud de las personas necesitadas de cuidados, corriendo el riesgo de omitirse su condición humana, integra y compleja.

Por otro lado, las enfermeras muchas veces también centran su quehacer en el trabajo administrativo, actividad que es parte de la asistencia del paciente y que se relaciona estrechamente con el "cuidado humanizado". Pero, que al mismo tiempo, acaba alejando al profesional de su lado humano y sensible. Aspecto que provoca algunas controversias: ¿Cómo se podría cuidar a un individuo olvidando su humanidad?, pensar en el cuidado deshumanizado del que habla Watson parece insólito, dado que se realiza sin empatía, sin respeto, sin el cariño que merece el cuidar del otro. ${ }^{6}$ Álvarez y Román ${ }^{9}$ indican que "El ejercicio profesional del equipo de salud adolece actualmente de un proceso de deshumanización en la sociedad globalizada, en la cual prevalece un vacío espiritual y la cultura de la banalidad". Esta tendencia del mundo globalizado afecta seriamente el trabajo en salud y las relaciones humanas como ya se dijo, cabiéndole a la enfermera preservar el único objetivo del cuidado: una atención con calidad humana, buscando la restitución de la integridad física y mental, es decir, del ser humano en todas sus áreas.

\section{SEGUNDO ARGUMENTO}

Un aspecto que aleja muchas veces a la Enfermera del cuidado humanizado es el tecnologismo, con la llegada a los servicios de tecnologías cada vez más sofisticadas y de alta especificidad, que ayudan a salvar vidas, antes impensables, las enfermeras muchas veces dan mayor importancia a la tecnología; pareciera que entre más tecnologizado sea el cuidado "es mejor" para el paciente. Creencia errada si se analiza el trasfondo que implica el manejo de la tecnología blanda en el cuidado al ser humano, quien es un ser que experimenta dolor, angustia y ansiedad, agregándose a esta situación las dudas y miedos de los familiares; hechos y sentimientos que la enfermera no puede evadir ni ser indiferente alegando sobrecarga laboral o desarrollo de su productividad. Siendo necesario implementar 
estrategias que faciliten el acogimiento, la escucha, la educación y en definitiva operar el "Cuidar" 5 en los servicios de salud. Torres ${ }^{10}$ reporta que los usuarios valoran mucho los aspectos del cuidado relacionados con la comunicación efectiva, destacando el tiempo para la escucha, disponibilidad de mayor atención, calidez en el trato, entre otros.

Cerullo y $\mathrm{Cruz}^{11}$ sostienen que para tornar la comunicación satisfactoria, el profesional debe involucrarse y creer que su sola presencia es tan importante como la realización de procedimientos técnicos y que es capaz de disminuir situaciones de estrés. Es decir, que la relevancia de los profesionales de Enfermería por las técnicas, los procedimientos, el uso de equipos sofisticados en la recuperación y mantenimiento de la vida y salud de las personas, las actividades de supervisión u otros aspectos importantes del quehacer profesional, no debe colocar al ser humano como alguien que solo necesita de un experto en el manejo de aparatos y técnicas procedimentales. Es innegable que son importantes pero no puede reemplazar la entrega y el cariño hacia los pacientes, siendo estos componentes complementarios y no contrarios. "Las actitudes y la práctica de los cuidados es lo único que distingue a la enfermería de las contribuciones de otras disciplinas". ${ }^{2}$

El realizar un procedimiento técnico cumpliendo altos estándares de calidad, es otra manera de cuidar con respeto; pero desde la percepción de los usuarios, el hacerlo bien no es tan importante para ellos como hacerlo con entrega y empatía. Realizando un real encuentro con el "otro" qué, en la perspectiva de Levinas, citado por Urigibel-Uriz, ${ }^{13}$ significa el encuentro infinito entre él mismo y el otro. Un encuentro que nace de la esencia incompleta de los seres humanos, encuentro que nutre, en el que él mismo busca al otro y en donde los dos tratan de completarse aun siendo seres incompletables. La tecnología dura, como cualquier instrumento básico o sofisticado, en sí misma, no es negativa ni positiva, lo que le da un determinado significado es el modo de usarse y aplicarlo en el cuidado. Así siendo, el profesional que maneja la tecnología dura debe practicar los principios éticos de respeto $\mathrm{y}$ dignidad humana con la persona enferma, lo que implica la necesidad de desarrollar una atención que humanice la tecnología, enmarcando su práctica en modelos conceptuales que tengan valores humanistas, que permitan cuidar a las personas con sus problemas, en lugar de cuidar únicamente los problemas que presentan las personas. ${ }^{1}$ El uso de tecnología blanda dura, exige de la enfermera, habilidades de cuidado y habilidades especiales, siendo imperativo comenzar a desarrollar conocimientos nuevos que emergen del diario quehacer para formar un corpus de conocimiento propio, que ayude a la enfermera a mejorar su interrelación en el proceso del cuidado, nuevos retos para no dejarse llevar por la super-especialización y medicalización que fragmenta a la persona y dificulta la integración del cuidado enfermera-paciente, como un binomio. $^{13}$

\section{TERCER ARGUMENTO}

Enfermería es un arte y una ciencia en crecimiento, sin embargo, ciertas formas de organización vinculadas a los avances tecnológicos $\mathrm{y}$, sobre todo, la forma en que algunas personas lo aplican amenazan la profesión. En el marco del arte se encuentran la intuición, la sensibilidad y la sabiduría, que permiten tener una visión global y transversal de las necesidades del ser humano que se cuida. ${ }^{14}$

Abordando la enfermería desde la formación, la aparente carencia de contenidos filosóficos en los currículos académicos no sería el único factor deshumanizante que se deriva de la formación de los profesionales de enfermería. Los programas académicos están paralelamente acompañados por el denominado currículo oculto, el cual, no consta en el diseño ni objetivos de los cursos, de este no se tiene conciencia, asimismo, es inevitable y muchas veces incongruente con los objetivos del programa. ${ }^{16}$ Por otro lado, es difícil mantener los valores humanitarios en el acto de cuidar en las instituciones públicas de salud, en donde parece invisibilizarse los cuidados de enfermería por las labores biomédicas, pero las enfermeras deben mantener entonces las virtudes de comunicarse con el otro, ayudarlo y sostenerlo en esta difícil etapa de su vida. Para tal efecto, el profesional debe practicar los principios éticos de respeto $\mathrm{y}$ dignidad humana con la persona que cuida, priorizando sus problemas como un todo: cuerpo, alma y espíritu.

Vecilla ${ }^{15}$ refiere que los rasgos de un cuidado humanizado son el reconocimiento de la dignidad intrínseca en todo ser humano; la identificación de cada persona como un ser único, el reconocimiento de sus diversas dimensiones, es decir, su componente holístico, lo que implica el abandono de posturas reduccionistas que convierten al 
paciente en una patología, un tratamiento o un conjunto de signos y síntomas. Es necesario buscar estrategias que trasladen los valores humanistas a la aplicación de las tecnologías y que permitan aprovechar lo positivo de éstas, sin perder los valores humanos de los cuidados de enfermería. Contrariamente, es posible que la aplicación de tecnologías pueda generar situaciones adversas, esta situación pone a la enfermera frente al reto de investigar las formas y opciones de dar solución a los problemas; el desarrollo de la investigación en el cotidiano quehacer de la enfermera es mandatorio, si se plantea que "una enfermera requiere en su práctica de diferentes tipos de conocimiento, como el científico, el artístico, el ético y el personal". ${ }^{18}$ En concordancia con Gortner, citado por Sánchez, ${ }^{17}$ el mayor de estos desafíos es la utilización de los hallazgos de la investigación en la práctica.

\section{CONSIDERACIONES FINALES}

Entonces ¿Cómo se observa el uso de la tecnología en la asistencia al ser humano? La enfermera requiere tener una actitud antropológica de la vida, de lo humano y del cuidado frente al mundo, un compromiso moral con el ser cuidado, contribuyendo con el bienestar general, la promoción de las potencialidades y de la dignidad humana.

Desde la perspectiva tecnológica, la calidad de vida tiene mucha relación con el desarrollo de la tecnología, ${ }^{15}$ brinda instrumentos diagnósticos y de tratamiento precoz y oportuno, con alta precisión y menos impacto lesivo para los usuarios, siendo la enfermera la responsable de cómo utilizar la tecnología. La técnica y la tecnología cobran un nuevo significado dependiendo de cómo se utilicen, ya que la acción y el acto de las personas también ayudan a construir el significado social de la tecnología; si se hace uso indebido en el que se considera a la persona como objeto se potenciará la deshumanización, mientras que si la utilizan como un medio facilitaran el trato humano. ${ }^{16}$

Se debe elevar los niveles de educación profesionalizando la disciplina para lograr el máximo nivel académico, es decir, una educación formativa que integre los valores y principios humanos, buscando estrategias para desarrollar la investigación en el área asistencial, que se conjugue en "una triada inseparable de asistencia, investigación y educación" "17 usando paradigmas cuantitativos y cualitativos que permitan fortalecer el cuerpo de conocimientos de enfermería.

El factor tecnológico en sí mismo, puede potenciar la calidad de vida de las personas, pero también, puede mejorar o empeorar la calidad de vida de esa persona. Cuando la tecnología está al servicio de lo humano y de su desarrollo, puede decirse con certeza que ayuda a potenciar la calidad de vida, sucede lo contrario cuando lo humano se somete al poder de lo tecnológico, entonces la calidad de vida puede verse muy alterada e inclusive desdeñada. En consecuencia, es probable que la tecnología aumente la necesidad del contacto humano, fundamental para la confianza y colaboración. Todo ello conlleva a un gran respeto por la vida, la que constituye un valor inapreciable e insustituible, pero también representa la necesidad de reconocer la dignidad para vivirla y la práctica de valores morales, comprendiendo el significado de lo ético, más allá de lo superficial o cosmético que permitirá entender la finalidad de la vida humana. ${ }^{15}$ Como dice Levinas: "La visión no es una trascendencia, otorga una significación por la relación que hace posible". 13

Finalmente, Enfermería aún tiene mucho por hacer y mejorar, desde la formación en valores como la responsabilidad, la dignidad, la honestidad, la solidaridad, la cortesía, el sentido de pertenencia son cualidades que se deben fomentar en los educandos de enfermería, los cuales permitirán reconocer su sensibilidad y sus sentimientos, al ser más generosos, auténticos y humanos. Esta formación humanista de respeto y amor a las personas es la tendencia más profunda del desarrollo moral de la Enfermería.

El compromiso seguirá siendo arduo, los profesionales que forman esta disciplina deben ser perseverantes para hacer del quehacer algo valioso y como afirma Tolle: ${ }^{18}$ "hay que tener claro que nuestro presente es el único tiempo real, debemos vivirlo", sin dejar de lado la esencia del cuidado: la PERSONA HUMANA.

\section{Correspondencia:}

Olga Giovanna Valderrama Ríos

Correo electrónico: olgagiovanna@ hotmail.com

REFERENCIAS BIBLIOGRÁFICAS 
1. Rocha PK, Prado ML, Cabral PFA. Souza AIJ, Anders JC. El cuidado y la tecnología en las unidades de cuidados intensivos. Index Enferm [internet]. Jul-sep 2013 [citado sep 20 de 2014]; 22(3):[07 laudas]. Disponible en: http://dx.doi.org/10.4321/S1132$\underline{12962013000200009}$

2. Boykin A, Schoenhofer OS. Nursing as Caring: A Model for Transforming Practice [internet]. Boston (EEUU): Jones And Bartlett Publishers; jun 2013 [citado sep 16 de 2014]; [aproximadamente 50 laudas]. Disponible en: http://www.gutenberg.org/files/42988/42988h/42988-h.htm\#link2HCH0003

3. Quintero MC, Pinto N. Cuidado y Práctica de Enfermería: Espiritualidad y afecto en el Cuidado de Enfermería. Grupo de cuidado. Facultad de Enfermería. Universidad Nacional de Colombia. Bogotá. 2000, pp. 184-191.

4. Ariza OC, Caballero RD. Calidad del cuidado de enfermería al paciente hospitalizado [internet]. Bogotá (Colombia): Pontifica Universidad Javeriana; jul 2008 [citado jul 13 de 2013]; [101 laudas]. Disponible en: http://books.google.es/books?id=L0T4LiRFUQC\&pg=PA22\&dq=uso+de+tecnologia+en +enfermeria\&hl=es\&sa=X\&ei=7XUDVLaoJ9 imggSQtYKYAw\&ved=0CEcQ6AEwBw\#v= onepage\&q\&f=false

5. Arredondo-Gonzales CP, Siles-Gonzales J. Tecnología y humanización de los cuidados. Una mirada desde la teoría de las relaciones interpersonales. Index Enferm [internet]. Enemar 2009 [citado sep 10 de 2014]; 18(1):[aproximadamente 08 laudas].Disponible en:http://scielo.isciii.es/scielo.php?pid=S1132 $\underline{-12962009000100007 \& \text { script=sci_arttext }}$

6. Watson J. Ponencia del primer congreso internacional de Enfermería [internet]. México: Universidad Autónoma de Chihuahua. 2007 [citado jun 04 de 2013]; [aproximadamente 03 laudas]. Disponible en: http://fen.uach.mx/index.php?/page/Semblanz a Jean_Watson.

7. Meleis A. Theoretical Nuring: development and progress [internet]. 3a. Ed. Philadelpia (EEUU): Lipincott-Williams \&Wilkins; 2005. Meleis A, Perspectivas en Enfermería (Reales
E, Traductor). Seminario de investigación II: Epistemología en Enfermería. Universidad Nacional de Colombia [citado may de 2013]; [aproximadamente 03 laudas]. Disponible en: http://es.calameo.com/read/000960602f3f95e3 $\underline{0 \mathrm{e} 8 \mathrm{~b} 5}$.

8. Aguirre RCD. Satisfacción laboral de los recursos humanos de enfermería, factores que afectan. Rev haba cienc méd [internet]. Octnov 2009 [citado may 13 de 2013]; 8(4):[aproximadamente 10 laudas]. Disponible en:

http://scielo.sld.cu/scielo.php?script=sci_artte $\underline{x t \& p i d=S 1729-519 X 2009000400021}$

9. Álvarez COM, Cisneros GR. Sensibilidad. Valor condicional del equipo de salud para el cuidado humano [internet]. Barcelona (España): Portales Médicos, 2008 [citado jun 13 de 2013]; [aproximadamente 10 laudas]. Disponible en: http://portalesmedicos.com/publicaciones/artic les/1187/1/sensibilidad-valor- Dcondicionaldel- equipo-de-salud-para-el-cuidadohumano.htlm

10. Torres CCC. Valoración de Aspectos Cualitativos del Cuidado de Enfermería en Pacientes Hospitalizados. Rev Cuid [internet]. 2010 [citado sep 15 del 2014]; 1(1): 10-18. Disponible en: http://cuidarte.udes.edu.co/index.php?option= $\underline{\text { com } \_ \text {content } \& \text { view }=\text { article } \& \text { id }=74 \& \text { Itemid }=9}$

11. Cerullo JASB. Cruz DALM. Raciocinio clínico y pensamiento Crítico. Rev Latino-am. Enfermagem [internet]. Ene-feb 2010 [citado ene de 2013]; 18(1): [06 laudas]. Disponible en:

http://www.scielo.br/pdf/rlae/v18n1/es_19.pdf

12. McFarland M. Wehbe-Alamah H. Leininger's Culture care diversity and universality a worldwide nursing theory [internet]. Third Ed. Ohama, Nebrasca (EEUU): Jones \& Bartlett Learing, 2012. [citado sep 03 de2014]; [aproximadamente 605 laudas]. Disponible en: http://books.google.com.pe/books?id=Py4EB AAAQBAJ\&pg=PA24\&lpg=PA24\&dq=(LEI NINGER;+McFARLAND,+2002;+2006)\&so urce $=$ bl\&ots $=\mathrm{Y}$ $\underline{\text { Y6lb06to\&sig=QD4w3Dc VgvIwMvDxzdVf }}$ zuxj_0\&hl=es419\&sa=X\&ei=FncvVMDwGte0yATMkIK4 
Cg\&ved=0CDIQ6AEwBA\#v=onepage $\& \mathrm{q}=(\mathrm{L}$ EININGER\%3B\%20McFARLAND\%2C\%20 2002\%3B\%202006)\&f=false

13. Irigibel-Uriz $X$. Nietzsche y Levinas, un marco de referencia para una enfermería transformadora. Index Enferm [internet]. 2007 [citado sep 16 de 2014]; 16(58): [aproximadamente 5 laudas]. Disponible en: http://scielo.isciii.es/scielo.php?script=sci_artt ext\&pid=S1132-12962007000300011

14. León RCA. Enfermería ciencia y arte del cuidado. Rev Cubana Enfermer [internet]. 2006 [citado sep 10 de 2014]; 22(4): [aproximadamente 3 laudas]. Disponible en: http://www.bvs.sld.cu/revistas/enf/vol22_4_06 lenf07406.htm

15. Vecilla SMAT. La Dignidad en los Cuidados de Salud. Ética de los Cuidados [internet]. Ene- jun 2008 [citado sep 18 de 2014]; 1(1): [aproximadamente 03 laudas]. Disponible en: http://www.index-

f.com/eticuidado/n1/et6759.php

16. Escudero RB. Humanismo y tecnología en los cuidados de enfermería desde la perspectiva docente [internet]. España: Fundación Dialnet, Universidad La Rioja. 2001 [citado sep 18 de 2014]. Disponible en: http://dialnet.unirioja.es/servlet/articulo?codig $\underline{\mathrm{o}=622314}$

17. Sánchez HB. La investigación en enfermería un problema de cuidado [internet]. Bogotá (Colombia): Biblioteca digital, Universidad Nacional de Colombia. 2001 [citado sep 14 de 2014]- Disponible en: http://www.bdigital.unal.edu.co/20333/1/1643 8-51322-1-PB.pdf

18. Tolle E. El poder del ahora: Un camino hacia la realización personal. USA: Norma, 2008 [citado agos 22 de 2014]; [aproximadamente 144 laudas]. Disponible en: http://academic.uprm.edu/dpesante/docsapicultura/elpoderdelahora.PDF 